\title{
ÜBER DAS RÄUMLICHE ACHTECK
}

\author{
WELCHES DIE SCHNITTPUNKTE DREIER OBERFLÄCHEN
}

ZWEITER ORDNUNG BILDEN

VON

\section{H. DOBRINER}

in FRANKFURT a. $M$.

Hesse beweist $\operatorname{im} 26^{\text {ten }}$ Bande von Crelles Journal (p. I 52) den Satz:

》Wenn die acht Schnittpunkte dreier, Oberflächen zweiter Ordnung mit o, 1, 2, 3, 4,5,6,7 bezeichnet werden, so liegen die vier Punkte

$$
(\text { OI } 2,45),(\text { ㄷ } 6,34),(745, \text { I } 2),\left(75^{6}, 23\right)
$$

oder, was dasselbe ist, die Schnittlinie (745, O I2) und die Punkte (o 6,34$)$ und $\left(75^{6}, 23\right)$ in einer und derselben Ebene.) ${ }^{1}$

Dieser Satz enthält eine Erweiterung des Pascal'schen Theorems." Liegen nämlich von den acht Schnittpunkten sechs und zwar die Punkte 1, 2 ...6 in einer Ebene $E$ (also auf einem Kegelschnitt), so sind die Punkte (O 6,34$)$ und $\left(75^{6}, 23\right)$ identisch mit den Punkten $(16,34)$ und $(56,23)$

1 An der citirten Stelle und vorher Bd. 20, p. 285-308, behandelt Hesse die obige Eigenschaft associirter Punkte synthetisch, später analytisch in den nachgelassenen Arbeiten Bd. 85, p. 304-316 und Bd. 99, p. I10-127.

${ }^{2}$ Vgl. HEsse: Note über die acht Schnittpunkte dreier Oberfächen zweiter Ordnung. Crelles Journ. Bd. 73, p. 37 I.

Acta mathematica. 12. Imprimé le 16 mai 1889. 
der Ebene $E$, während die Schnittlinie (745, о \2) den Punkt (45, 12) enthält. Die drei Punkte $(12,45),(23,56),(34,6$ I $)$ müssen demnach, weil sje gleichzeitig der Ebene $E$ und der durch den Hesse'schen Satz festgestellten Ebene angehören, auf einer Gieraden liegen. Diese ist aber keine andere als die Pascal'sche Linie des Sechsecks, dessen aufeinander folgende Ecken die Punkte I, 2..6 sind.

Bezeichnet man mit dem Symbol

$$
H(\text { O } 2 \cdot 3 \cdot 456 \cdot 7)
$$

diejenige Ebene, welche durch die Schnittlinie

$$
\text { (OI } 2,456)
$$

und die Punkte

$$
\left(3^{2}, 765\right),(34,701)
$$

bestimmt ist, ferner im Grenzfalle, wenn die Punkte 1, 2 .6 in einer Ebene liegen, mit dem Symbol

$$
P(\text { I } 23456)
$$

diejenige Pascal'sche Linie, auf welcher die Punkte

$$
(\text { I } 2,45),(23,56),(34,61)
$$

liegen, so kann man das vorhergehende dahin zusammenfassen, dass die Ebene $H\left(\mathrm{Ol}_{2} \cdot 3 \cdot 457.6\right)$ im Grenzfalle die Pascal'sche Linie $P\left(\right.$ I $_{2456)}$ enthält.

Hierdurch ist zwischen $H$-Ebenen und Pascal'schen Linien eine Correspondenz hergestellt. Es erschien mir nun als eine lohnende Aufgabe zu untersuchen, ob den Eigenschaften der Pascal'schen Linien, die sich in den Sätzen von Steiner, Kirkman, Cayley und Salmon aussprechen, auch analoge Eigenschaften der entsprechenden $H$-Ebenen gegenüberstehen. Von diesem Gesichtspunkte aus ist meines Wissens die räumliche Figur, welche von den Schnittpunkten dreier Flächen zweiter Ordnung gebildet wird, noch nicht behandelt worden.

Es sei mir gestattet, hier die Sätze übersichtlich zusammenzustellen welche im Grenzfalle in die vorhin erwähnten übergehen. 
Über die acht Schnittpunkte dreier Oberflächen zweiter Ordnung.

I.) Nach Steiner schneiden sich die drei Pascal'schen Linien

$$
P(\text { г } 23456), P(\text { 1 } 43652), P(\text { I63254), }
$$

oder, was dasselbe ist, die drei Linien

$$
P(123456), P(325416), P(52 \text { 1 436) }
$$

in einem Punkte. Für die $H$-Ebenen gelten die beiden folgenden Sätze:

a) Die drei Ebenen

$$
H(\text { O } 2 \cdot 3 \cdot 457 \cdot 6), H(\text { OI } 4 \cdot 3 \cdot 657 \cdot 2), H(\text { o } 6.3 \cdot 257 \cdot 4)
$$

schneiden sich in einer geraden Linie. (\$ 6.)

b) Die drei Ebenen

$$
H(\text { I } 2 \cdot 3 \cdot 457 \cdot 6), H(032 \cdot 5 \cdot 4 \text { I } 7 \cdot 6), H(052 . \text { I } \cdot 437 \cdot 6)
$$

schneiden sich in einem Punkte der Ebene (670). (\$ 7.)

2.) Dem Kirkman'schen Satze, dass sich die drei Linien

$$
P\left(624 \text { I53) } P\left(46315^{2}\right), P\left(64135^{2}\right) \equiv P(264135)\right.
$$

in einem Punkte schneiden, steht der folgende gegenüber:

a) Die sechs Ebenen

$$
\begin{aligned}
& H(062 \text {. 4 . I } 57 \text {.3), H(046.3. 1 } 57 \text {. 2), H(064. I . 357.2), } \\
& H(\mathrm{O} 26 \text {. 4. I 37.5) } H(264 \text {. I . } 357.0), H(026.4 \text {. I } 35 \text {. 7) }
\end{aligned}
$$

schneiden sich in einem Punkte. ( $(8$.

Es entspricht nur einer Vertauschung der Ziffern 2, 3 mit resp. 4, 6, wenn man einen Kirkman'schen Punkt als den Schnittpunkt der Linien

$$
P(\text { 1 } 23456), P(\text { 163245) } P(\text { I 56342) } \equiv P(124365)
$$

definirt. Für die $H$-Ebenen hingegen besteht das Analogon in einem neuen Satze:

b) Die vier Ebenen

$$
\begin{gathered}
H(\text { o } 2 \cdot 3 \cdot 457 \cdot 6), H(\text { oI } 6 \cdot 3 \cdot 247 \cdot 5), H(\text { OI } 5 \cdot 6 \cdot 347 \cdot 2), \\
H(\text { oI } 2 \cdot 4 \cdot 367 \cdot 5)
\end{gathered}
$$

schneiden sich in einem Punkte der Ebene (346). ( $\$$ Io.) 
3.) Vertauscht man in den zuerst angegebenen Symbolen der Pascal'schen Linien, welche sich in einem Kirkman'schen Punkte schneiden, die Ziffern 2, 4,6 cyklisch, so erhält man drei Punkte, die (nach CAYLEY und Salmon) auf einer Geraden liegen. Ein wörtlich gleich lautender Satz besteht für die durch Satz 2. a) definirten Punkte. (§ 9.)

Mir ist es zwar nicht gelungen, auch zu den Steiner'schen Linien und zu den Salmon'schen Punkten in der räumlichen Figur entsprechende Gebilde zu finden. Ich bezweifle aber nicht, dass sich jeder Satz über Pascal'sche Linien als ein Grenzfall eines oder mehrerer allgemeineren, die $H$-Ebenen betreffenden Sätze ergeben wird.

\section{$\S \mathrm{I}$.}

Zu den Hesse'schen synthetischen Beweisen seines Satzes füge ich einen neuen hinzu, der noch weitere Eigenschaften der $H$-Ebenen erschliessen soll. ${ }^{1}$

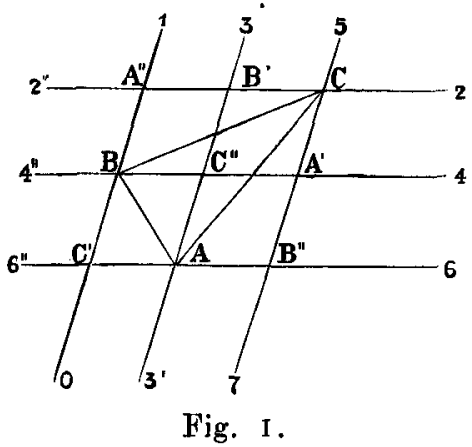

Ich ziehe die Geraden (OI) und (75) und lege von den Punkten 2, 4,6 aus die Transversalen, welche jene Geraden schneiden. Diese Transversalen $\left(22^{\prime \prime}\right),\left(44^{\prime \prime}\right),\left(66^{\prime \prime}\right)$ bestirnmen ein Hyperboloid, welches durch die siehen Punkte $0,1,2,4,5,6,7$ geht; wegen der Fundamental-Eigenschaft der Schnittpunkte dreier Flächen zweiter Ordnung muss es dann auch den achten Punkt 3 enthalten. Von diesem lässt sich also eine Linie (33') ziehen, welche die drei Transversalen schneidet.

Man hat dann zweimal drei Erzeugende des Hyperboloids mit neun Schnitt. punkten, die ich in folgender Weise bezeichne:

$$
\begin{array}{lll}
\text { (O I , 22 2") }=A^{\prime \prime}, & \left(3^{\prime} 3,22^{\prime \prime}\right)=B^{\prime}, & \left(75,22^{\prime \prime}\right)=C, \\
\text { (OI } \left., 44^{\prime \prime}\right)=B, & \left(3^{\prime} 3,44^{\prime \prime}\right)=C^{\prime \prime}, & \left(75,44^{\prime \prime}\right)=A^{\prime}, \\
\text { (O I } \left., 66^{\prime \prime}\right)=C^{\prime}, & \left(3^{\prime} 3,66^{\prime \prime}\right)=A, & \left(75,66^{\prime \prime}\right)=B^{\prime \prime} .
\end{array}
$$

1 Man vgl. die Anmerkung zu $\$ 6$. 
Über die acht Schnittpunkte dreier Oberfächen zweiter Ordnung.

Die Gerade $B C$ ist die Schnittline (O I 2,457 ),

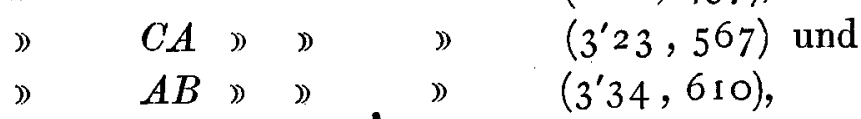

folglich liegt auf $C A$ der Punkt $(23,567)$ und auf $A B$ der Punkt $(34,6$ Io). Mithin sind die Schnittlinie (O I 2, 457) und die beiden Punkte $(23,567)$ und $(34,6$ Io), - wie es der Hesse'sche Satz behauptet, - in einer Ebene enthalten, nämlich in der Ebene $A B C$.

Sucht man in einer beliebigen Ebene $E$ die Spuren der Geraden und Ebenen der vorstehenden Figur, so findet man durch die sechs Erzeugenden des Hyperboloids sechs Punkte eines Kegelschnitts $(a, b, c, d, e, f)$ bestimmt, und durch die Ebene $A B C$ eine Linie $P$, welche die drei Punkte $(a b, d e),(b c, e f),(c d, f a)$ enthält. Damit ist ein einfacher Beweis fur das Pascal'sche Theorem gegeben. Man kann auch umgekehrt von sechs Punkten I , 2 . 6 eines Kegelschnitts ausgehen und zur Construction der Figur zwei beliebige von $\mathrm{I}$ und 5 aus in den Raum gezogene windschiefe Linien (10) und (57) zu Hülfe nehmen.

$$
\S 2 .
$$

Das Symbol

$$
H(\mathrm{O} 2 \cdot 3 \cdot 456 \cdot 7)
$$

soll die $H$-Ebene darstellen, welche durch die Linie (o1 2,456$)$ und die beiden Punkte $(32,567)$ und $(34,107)$ bestimmt ist.

Vertauscht man o mit I, oder 5 mit 6, oder 2, I, o mit resp. $4,5,6$, so bleiben die Elemente, welche die $H$-Ebene definiren, dieselben. Für jede $H$-Ebene sind mithin 8 Symbole vorhanden:

$$
\begin{aligned}
& H(\text { о I } 2.3 .456 .7), H(\text { I } 2.3 .456 .7), H(\text { I 2.3.465.7) } H(\text { I O2.3.465.7), } \\
& H(654.3 .2 \text { I0.7 }), H(654.3 .201 .7), H(564.3 .2 \text { 10.7), H(564.3.201.7). }
\end{aligned}
$$

Da nun die Zahl aller möglichen Symbole 8 ist, so schliessen wir:

Es giebt $\mid \underline{z}=5040 \mathrm{H}$-Ebenen für die Schnittpunkte dreier gegebenen Oberflächen zweiter Ordnung. 
Nenne ich (OI 2,345) eine Linie $L$ und (OI, 234) einen Punkt $V$, so ist die Zahl aller Linien $L$ gleich 280 und die Zahl aller Punkte $V$ gleich 560. Auf jeder Linie $L$ liegen 6 Punkte $V$ und durch jeden Punkt $V$ gehen 3 Linien $L$; so liegen z. B. auf (or 2, 345) die Punkte:

$$
(\mathrm{I} 2,345),(20,345),(\text { O I }, 345),(45, \text { OI } 2),(53 \text {, O I } 2),(34, \text { OI } 2) \text {; }
$$

durch (OI, 234) gehen die Linien:

$$
(501,234),(601,234),(701,234) \text {. }
$$

Jede $H$-Ebene enthält eine Linie $L$ und durch jede Linie $L$ gehen I $8 H$ Ebenen, denn aus $H$ (OI 2.6.345.7) erhält man weitere durch (OI 2, 345) gehende $H$-Ebenen, wenn man folgende Vertauschungen gesondert oder gleichzeitig vornimmt:

a) 2 mit o oder mit $I$,

b) $3 \gg 4 \% 5$,

c) $6 \gg 7$.

In jeder $H$-Ebene liegen 8 Punkte $V$, von welchen 6 der Linie $L$ angehören; diese nenne ich Punkte der zweiten Art, die beiden andern Punkte der ersten Art.

Durch jeden Punkt $V$ gehen 3 Linien $L$, und durch jede derselben $18 H$-Ebenen; es gehen also durch jeden Punkt $V 54 H$-Ebenen, für welche er ein Punkt der zweiten Art ist. Für I 8 andere $H$-Ebenen ist er ein Punkt der ersten Art; z. B. ist (OI , 234) ein Punkt der ersten Art für $H(345.0 .167 .2)$ und diejenigen $H$-Ebenen, deren Symbole man durch folgende Vertauschungen erhalt:

a) 2 mit 3 oder mit 4 ,

b) $5 \gg 6 \gg 7$,

c) $0 \gg \mathrm{I}$.

$\S 3$.

Zieht man die Verbindungslinien zwischen den acht $V$-Punkten der Ebene $H$ (OI2.3.456.7), so erhält man ausser $L$ I 3 Linien, die ich in fünf Gruppen ordne: 
Uber die acht Schuittpunkte dreier Oberflächen zweiter Ordnung.

(a)

$$
(32,567)-(34,107)
$$

(b)

$$
\begin{aligned}
& \left(3^{2}, 5^{67}\right)-\left(5^{6}, \text { O I } 2\right),(34, \text { I О })-\left(\text { O I }, 45^{6}\right) \text {; } \\
& (32,567)-\left(20,45^{6}\right),(32,567)-(21,456) \text {, } \\
& (34, \text {, І } 7)-(45 \text {, о I } 2),(34, \text { O } 7)-(46 \text {, о } 2) \text {; } \\
& \left(3^{2}, 5^{67}\right)-\left(\text { OI }, 45^{6}\right),(34, \text { O I } 7)-\left(5^{6} \text {, O I } 2\right) \text {; } \\
& \left(3^{2}, 5^{6} 7\right)-(45,012),(32,567)-(46, \text { O I } 2), \\
& (34, \text { O I } 7)-(20,456),(34, \text { OI } 7)-(21,456) \text {. }
\end{aligned}
$$

(e)

Durch die beiden Punkte der Gruppe (a) geht nur die eine Ebene $H$ (OI2.3.456.7), ebenso durch jedes Paar der Gruppe (b). Hingegen gehen durch $(32,567)$ und $(20,456)$ die Ebenen

$$
\begin{aligned}
& H(\text { O } 2.3 \cdot 456.7), \\
& H(3 \text { I 2.0.756.4); }
\end{aligned}
$$

ebenso gehen durch jedes Paar der Gruppe (c) $2 H$-Ebenen.

Durch $(32,567)$ und (or , 456) gehen:

$$
\begin{aligned}
& H\left(\text { OI 2.3.456.7) } H\left(23 \text { O. . } 75^{6.4}\right),\right. \\
& H(\text { O } 3.2 .456 .7), H(23 \text { I.0.756.4); }
\end{aligned}
$$

durch das andere Paar aus (d) gehen gleichfalls $4 H$-Ebenen:

Durch $(32,567)$ und $(45$, Or 2$)$ gehen:

$$
\begin{aligned}
& H(\mathrm{O} 12 \cdot 3 \cdot 456.7), H(\mathrm{I} 23.4 \cdot 567.0), \\
& H(\mathrm{O} 2.3 .457 .6), H(\mathrm{O} 23.4 .567 . \mathrm{I}) .
\end{aligned}
$$

Nennt man die Verbindungslinie zweier Punkte der Gruppe (e) eine Linie $A$, so dass $A$ definirt ist als die Verbindungslinie von $(32,567)$ und $(45,210)$ oder zweier anderen V-Punkte, die aus diesen hervorgehen, wenn $\operatorname{man} \mathrm{O}, \mathrm{I} \ldots 7$ durch eine beliebige Permutation ersetzt, so kann man den Satz aussprechen:

Jede $H$-Ebene enthalt 4 Linien $\Lambda$, und durch jede Linie $\Lambda$ gehen $4 H$-Ebenen.

Acta mathematica. 12. Imprimé le 21 mai 1889. 
Die Zahl der $A$ muss also gleichfalls 5040 sein. In der That gehen von jedem Punkte $V$ r 8 Linien $\Lambda$ aus, so dass die Zahl derselben

ist.

$$
560.18: 2=5040
$$

)Die $5040 H$-Ebenen und die 5040 A-Linien bilden eine räumliche Configuration von der Art, dass jede Ebene vier Linien enthält, und durch jede Linie vier Ebenen hindurch gehen.»

$$
\S 4 \text {. }
$$

Die vorhergehende Zusammenstellung der (wesentlich) verschiedenen Paare von $V$-Punkten, von welchen jedes mindestens in einer $H$-Ebene liegt, wird es im einzelnen Falle ermöglichen zu entscheiden, ob sich durch zwei gegebene $V$-Punkte überhaupt $H$-Ebenen legen lassen, und wie gross etwa die Anzahl derselben ist.

Liegen vier V-Punkte in einer Ebene, und gehen durch zwei Gegenseiten des von ihnen gebildeten Vierecks je 2 (oder 4) $H$-Ebenen, so hat der Schnittpunkt der Gegenseiten die besondere Eigenschaft, dass sich in ihm 4 (oder mehr) $H$-Ebenen schneiden.

Ich führe einige Falle dieser Art an, ohne entscheiden zu wollen, ob sie die einzigen, überhaupt möglichen sind.

Nach $\$ 3$ liegen die Linie (d) zwischen $(23,567)$ und (OI , 456) und die Linie (e) zwischen $(34,017)$ und $(2 \mathbf{1}, 456)$ in der Ebene $H$ (O I 2.3.456.7); zugleich gehen durch jede der beiden Linien überdies noch $3 H$-Ebenen; folglich treffen sich in ihrem Schnittpunkt im ganzen $7 H$-Ebenen.

Betrachtet man ferner die Ebene, welche durch den Punkt (5) und die Linie (012, 034) bestimmt ist; sie enthïlt 22 V.Punkte und unter diesen die vier

$$
(\text { I } 2,034),(34, \text { O I } 2),(05,167),(05,367) \text {. }
$$

Durch die Linie $A_{1}=(12,0.34)-(05,167)$ gehen die Ebenen

$$
\begin{aligned}
& H_{0}(340.5 .2 \mathrm{I} 6.7), H_{2}(305.2 .167 .4), \\
& H_{1}(340.5 .217 .6), H_{3}(405.2 .167 .3),
\end{aligned}
$$


Über die acht Schuittpunkte dreier Oberflächen zweiter Ordnung.

und durch die Linie $A_{2}=(34$, O I 2$)-(05,367)$ die Ebenen

$$
\begin{aligned}
& H^{0}(\text { I } 20.5 \cdot 436.7), H^{\prime \prime}(105 \cdot 4 \cdot 367.2), \\
& H^{\prime}(\text { I } 20.5 \cdot 437.6), H^{\prime \prime \prime}(=05.4 \cdot 367.1) ;
\end{aligned}
$$

in dem Schnittpunkt von $A_{1}$ und $A_{2}$ treffen sich also $8 H$-Ebenen. Liegen die Punkte I, 2 .6.6 in einer Ebene $E$, so fällt der Schnittpunkt von $A_{1}$ und $A_{2}$ in den Punkt ( 2,34 ), folglich müssen durch diesen die acht Linien gehen, in welchen $E$ von 'den Ebenen $H_{0}, H^{0}, \ldots, H^{\prime \prime}$ geschnitten wird.

Nun sind aber, wie man leicht erkennt, die Durchschnitte von $E$ mit

$$
H_{0}, H^{0}, \quad H_{2} \quad, \quad H^{\prime \prime}, H_{3} \quad, \quad H^{\prime \prime \prime}
$$

die Linien

$3 \%$

$$
\text { (34), (1 2), } P(352 \text { 164), } P(\text { I } 54362), P(452 \text { I } 63), P(25436 \text { I }) \text {, }
$$

während die Ebene $E$ von $H_{1}$ und $H^{\prime}$ in unbestimmten, aber jedenfalls durch (12, 34) gehenden Linien geschnitten wird. Wir können also schliessen, dass die angeführten Pascal'schen Linien (von denen die beiden letzten identisch sind) durch den Punkt (1 2,34) gehen, ein Ergebniss, das auch unmittelbar aus der Bedeutung der Symbole $P$ hervorgeht.

Zum Schluss betrachte ich noch die Ebcne durch die beiden vom Punkte (o) ausgehenden Linien (O.12, 034) und (O. 5,036$)$ und in derselben die vier V.Punkte

$$
(\text { I } 2,034),(15,036),(34, \text { O I } 2),(36, \text { O } 15) \text {. }
$$

Durch die Verbindungslinie der beiden ersten gehen

$$
H(034.5 .127 .6) \text { und } H(036.2 .157 .4) \text {, }
$$

und durch die Verbindungslinie der beiden letzten

$$
H(\text { O } 2.6 .347 \cdot 5) \text { und } H(\text { O } 5 \cdot 4 \cdot 367.2) \text {. }
$$

Im Schnittpunkt der beiden Verbindungslinien treffen sich also $4 H$-Ebenen. 


\section{$\S 5$.}

Neben den Punkten $V$ enthält jede $H$-Ebene noch eine Reihe anderer Punkte, die ebenfalls unmittelbare, wenn auch nicht gleich einfache, Beziehungen zu dem Systeme der Punkte o, 1..7 haben.

Um sie zu finden, bediene ich mich einer Methode, die schon Hesse beim ersten Beweise ${ }^{1}$ seines Satzes angewandt hat, und die sich auf die von ihm gefundene Eigenschaft der Schnittpunkte dreier Flächen zweiter Ordnung stützt, dass sie, auf beliebige Art in zwei Gruppen von je vier geteilt, als die Ecken zweier Polartetraeder in Bezug auf eine (durch sie bestimmte) Fläche zweiter Ordnung angesehen werden konnen.

a) Es seien $(0,1,2,3)$ die Ecken des einen, und $(4,5,6,7)$ die Ecken des andern Tetraeders; ich suche den Pol der Ebene $H(O$ I 2.7.356.4) in dem durch jene bestimmten Polarsystem. Die reciproke Polare der Linie (OI 2, 356) geht durch die Punkte (3) und (47, O 2); die Polare von $(27,456)$ ist die Ebene durch den Punkt (7) und die Linic (O I 3,456$)$; die Polare endlich von $(73$, OI4) ist die Ebene durch $(012,456)$ und $(23,567)$ d. h. die Ebene $H(012 \cdot 3 \cdot 456.7)$.

Es besteht mithin der Satz:

»Der Punkt $p$, in welchem die Linie $[(3)-(47$, O 12$)]$ die Ebene

$\left[(7)-\left(\right.\right.$ O I $\left.\left.3,45^{6}\right)\right]$ schneidet, liegt in $H($ O I 2.3 .456 .7$\left.).\right)$

Ein zweiter Punkt $p$ in $H$ ist der Durchbohrungspunkt der Linie $[(3)-(27,564)]$ mit der Ebene $[(7)-(563,201)]$.

b) Ich polarisire ferner die Ebene $H$ (OI7.3.426.5) in Bezug auf die Tetraeder (0127) und (3456). Die reciproke Polare von (017, 426) ist die Linie $[(2)-(35$, o I 7$)]$, die Polare von $(34$, o I5) die Ebene $[(56)-(27,346)]$ und die Polare von $(37,265)$ die Ebene durch $(012,456)$ und (34, Or 7 ), das ist die Ebene $H$ (OI 2.3.456.7):

)Der Punkt $q$, in welchem die Linie [(2)- (35, o I 7$)]$ die Ebene

$\left[\left(5^{6}\right)-(27,346)\right]$ trifft, liegt in $\left.H\left(\mathrm{O}_{2} 2.3 .456 .7\right).\right)$

'Crelles Journal, Bd. 20, p. 305. 
In derselben $H$-Ebene giebt es noch drei Punkte $q$, deren Symbole man findet, wenn man zuerst 2,1 , o mit resp. 4, 5, 6, und dann 5 mit 6 vertauscht.

c) Es werde $H($ O I 5.7.326.4) polarisirt in Bezug auf die Tetraeder (OI23) und (4567). Die reciproke Polare von (OI5, 326) ist die Linic $[(23,467)-($ O I, 457$)]$, die Polare von $(57,264)$ die Ebene $[(46)-(57$, o I 3$)]$ und dic Polare von $(37$, or 4$)$ die Ebene $[(012,456)-(23,567)]$ d. i. $H$ ( O I $2 \cdot 3 \cdot 456 \cdot 7)$.

)Der Punkt $r$, in welchem die Linie $[(23,467)-(01,457)]$

die Ebene $[(46)-(57$, or 3$)]$ trifft, liegt in $H($ o $12 \cdot 3 \cdot 456.7)$.)

Durch dieselben Vertauschungen wie in b) findet man dic Symbole der drei übrigen Punkte $r$, die noch in $H$ liegen.

d) Schliesslich gelangt man, indem man $H(250.7 \cdot 314.6)$ in Bezug auf (O 27 ) und (3456) polarisirt, zu dem Satze:

${ }_{1}$ Der Punkt $s$, in welchem die Linic $[(17,346)-(56,207)]$

die Ebene $[(2 \mathrm{I})-(35,027)]$ trifft, liegt in $H($ o I $2.3 \cdot 456.7)$.

Die Zahl der Punkte $s$ ist 8 ; die Symbole der übrigen erhält man, wenn man gesondert und gleichzeitig o mit I, 5 mit 6, und o, I , 2 mit resp. $5,6,4$ vertauscht.

\section{$\S 6$.}

Wir gehen wieder auf die Figur des $\S$ I zurück, um neue Beziehungen zwischen $H$-Ebenen kennen zu lernen. ${ }^{1}$

Die Ebene $A B C$ enthielt die Linie $(012,457)$ und die Punkte $(32,567)$ und $(34,60 \mathrm{I})$; es ist mithin

$$
A B C=H\left(\mathrm{O}_{2} 2 \cdot 3 \cdot 457 \cdot 6\right)
$$

1 In diesem Paragraphen sind nur die Resultate der Hesse'schen Abhandlung Über das geradlinige Sechseck auf dem Hyperboloid (Crelles Journal, Bd. 24, p. 4043) reproducirt und für die Configuration der $H$-Ebenen verwerthet. Den Übergang aus dem Raum in die Ebene bewerkstelligt Hesse, indem er die Erzeugenden des Hyperboloids sich unendlich einer Ebene nähern lässt und dadurch zu einem Brianchon'schen Sechseck gelangt. Die oben in $\S$ I hergestellte Beziehung zu einem Pascal'schen Sechseck verdient, wie ich glaube, den Vorzug vor jenem Grenzübergange. 
Vertauscht man die Ziffern 2, 4,6 cyklisch, so machen sowohl die Buchstaben $A B C$ als ihre Indices die cyklische Vertauschung mit; demnach ist

$$
\begin{aligned}
& A B C=H(\text { OI } 2 \cdot 3 \cdot 457 \cdot 6)=H, \\
& A^{\prime} B^{\prime} C^{\prime \prime}=H(\text { or } 4 \cdot 3 \cdot 657.2)=H^{\prime}, \\
& A^{\prime \prime} B^{\prime \prime} C^{\prime \prime}=H(\text { ol } 6.3 .257 .4)=H^{\prime \prime}
\end{aligned}
$$

Ferner ist $A^{\prime} A^{\prime \prime}=\left(\mathrm{OI}_{4}, 257\right), A^{\prime \prime} A=\left(0 \mathrm{1} 6,323^{\prime}\right)$ und $A A^{\prime}=\left(756,343^{\prime}\right)$, mithin

$$
\begin{aligned}
& A A^{\prime} A^{\prime \prime}=H(\text { or } 4 \cdot 3 \cdot 257.6)=H_{i}, \\
& B B^{\prime} B^{\prime \prime}=H(\text { or } 6.3457 .2)=H_{3}, \\
& C C^{\prime} C^{\prime \prime}=H(\text { O } 2.3 .657 .4)=H_{3} .
\end{aligned}
$$

Nun ist

$$
\begin{aligned}
& B C=(\text { O } 12,457), \\
& B^{\prime} C^{\prime \prime}=\left(\text { O } 12,3^{6} 3^{\prime}\right), \\
& B^{\prime \prime} C^{\prime \prime}=\left(457,3^{6} 3^{\prime}\right)
\end{aligned}
$$

folglich schneiden sich

$$
\begin{aligned}
& B C, B^{\prime} C^{\prime \prime}, B^{\prime \prime} C^{\prime \prime} \quad \text { in dem Punkte } p_{1}=\left(0.2,3^{\prime} 36,745\right) \text {, } \\
& \left.C A, C^{\prime \prime} A^{\prime}, C^{\prime \prime} A^{\prime \prime} \quad 》 \quad \text { 》 } \quad p_{2}=\text { (OI } 4,3^{\prime} 3^{2}, 765\right) \text {, } \\
& A B, A^{\prime} B^{\prime}, A^{\prime \prime} B^{\prime \prime} \quad 》 \quad \text { » } p_{3}=\left(\cup 16,3^{\prime} 34,725\right) \text {. }
\end{aligned}
$$

Diese drei Punkte gehören aber zugleich den drei Ebenen $H, H^{\prime}, H^{\prime \prime}$ an, folglich müssen sie in einer Geraden $S t=\left(p_{1} p_{2} p_{3}\right)$ liegen.

Ferner findet man, dass sich schneiden

$$
\begin{aligned}
& A^{\prime} A^{\prime \prime}, B^{\prime} B^{\prime \prime}, C^{\prime} C^{\prime \prime} \text {, in dem Punkte } p=\left(\text { O I } 4,3^{\prime} 36,75^{2}\right) \text {, } \\
& A^{\prime \prime} A, B^{\prime \prime} B, C^{\prime \prime} C \quad 》 \quad \text { » } \quad p^{\prime}=\left(016,3^{\prime} 32,754\right) \text {, } \\
& \left.A A^{\prime}, B B^{\prime}, C C^{\prime \prime} \quad 》 \quad\right) \quad p^{\prime \prime}=\left(\text { O I } 2,3^{\prime} 34,75^{6}\right) \text {. }
\end{aligned}
$$

Diese drei Punkte müssen gleichfalls, weil sie den drei Ebenen $H_{1}, H_{2}, H_{3}$ angehören, auf einer Geraden $S t_{0}=\left(p p^{\prime} p^{\prime \prime}\right)$ liegen. 
Hiermit ist der Satz bewiesen:

„Die drei Ebenen

$$
\begin{aligned}
& H=H(\text { O } 2 \cdot 3 \cdot 457.6), \\
& H^{\prime}=H(\text { o } 4 \cdot 3 \cdot 657 \cdot 2), \\
& H^{\prime \prime}=H(\text { O } 6.3 .257 .4)
\end{aligned}
$$

schneiden sich in einer geraden Linie $S t$.

Die Symbole der Ebenen $H_{1}, H_{2}, H_{3}$, die sich in der Geraden $S t_{0}$ schneiden, gehen aus den vorstehenden durch Vertauschung von 2 mit 4 hervor.

Im Grenzfalle, wenn die Punkte I , 2 .6 in einer Ebene $E$ liegen, wird nach den Bemerkungen in der Einleitung die Ebene $E$

$$
\begin{aligned}
& \text { von } H \text { in der Pascal'schen Linie } P(123456) \text {, } \\
& \text { » } H^{\prime} \gg, \gg P(\text { I } 43652) \text {, } \\
& \text { 》 } \left.H^{\prime \prime}\right) \text { 》 》 } P(163254)
\end{aligned}
$$

geschnitten.

Aus unserm Satze folgt dann, dass sich diese drei Pascal'schen Linien in einem Punkte treffen. In der That liefern sie, wie bekannt, einen Steiner'schen Punkt. Die Linien St sind also Analoga zu diesen Punkten der Pascal'schen Figur.

Die beiden den Linien $S t$ und $S t_{0}$ entsprechenden Punkte heissen Steiner'sche Gegenpunkte.

Die Ebenen (OI2), $\left(3^{\prime} 36\right),(754)$ sind sämmtlich Tangentialebenen des Hyperboloids und berühren dasselbe in den Punkten $A^{\prime \prime}, A, A^{\prime}$. Mithin ist

$$
\begin{array}{cccc}
p_{1} & \text { der Pol der Ebene } & H_{1}, \\
p_{2} & \cdots & » & H_{2}, \\
p_{3} & \cdots & \cdots & H_{3} .
\end{array}
$$

In gleicher Beziehung stehen die Punkte $p, p^{\prime}, p^{\prime \prime}$ zu den Ebenen $I I, H^{\prime}, H^{\prime \prime}$. Daraus folgt der Satz:

गDie Linien $S t$ und $S t_{0}$ sind reciproke Polaren in Bezug auf das Hyperboloid, das durch die Linien (OI), (57) und die Punkte $2,3,4,6$ bestimmt ist.)

Ihm entspricht in der Ebene der Hesse'sche Satz:

)Zwei Steiner'sche Gegenpunkte sind harmonische Pole in Bezug auf den Kegelschnitt, dem das Pascal'sche Sechseck eingeschrieben ist.» 
Man erkennt leicht, dass jede $H$-Ebene nur durch eine Linie St geht; es giebt also im ganzen 5040:3 = 1680 Linien $S t$.

\section{$\S 7$.}

Die Symbole der drei Pascal'schen Linien, welche den der Linie St entsprechenden Steiner'schen Punkt liefern, gehen auch aus $P$ ( I 23456) hervor, wenn man nicht 2, 4,6 sondern 1,3,5 cyklisch vertauscht. Es fragt sich nun, welche Beziehungen bestehen zwischen den drei Ebenen

$$
\begin{aligned}
H & =H\left(0_{1} 2.3 \cdot 457.6\right), \\
{ }^{\prime} H & =H\left(0_{32} 2.5 \cdot 4 \mathrm{I} 7.6\right), \\
{ }^{\prime} H & =H\left(0_{52} 2.437 .6\right) .
\end{aligned}
$$

Ich betrachte die drei Hyperboloide, welche die Construction dieser Ebenen vermitteln. Jedes von ihnen enthält die acht Punkte $0,1 \ldots 7$; sie unterscheiden sich aber durch die Geraden (ik), die ihnen als Erzeugende zugehoren; beiın ersten Hyperboloid sind es die Linien (or) und (75), beim zweiten (03) und (7 I), beim dritten (05) und (73).

Am deutlichsten werden die folgenden Figuren die Entstehungsart der Hyperboloide und die Lage der Ebenen $H=(A B C),{ }^{\prime} H=\left({ }^{\prime} A^{\prime} B^{\prime} C^{\prime}\right)$, $" H=\left(" A^{\prime \prime} B^{\prime \prime} C\right)$ veranschaulichen.

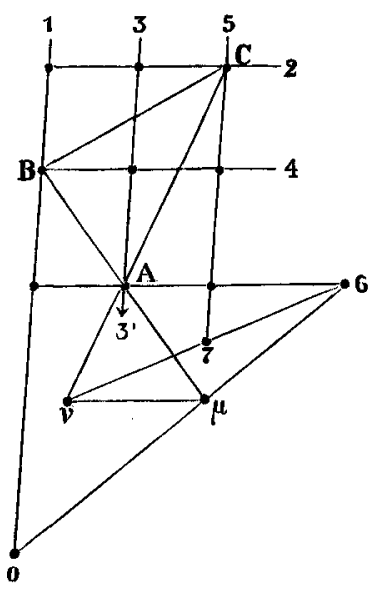

Fig. 2.

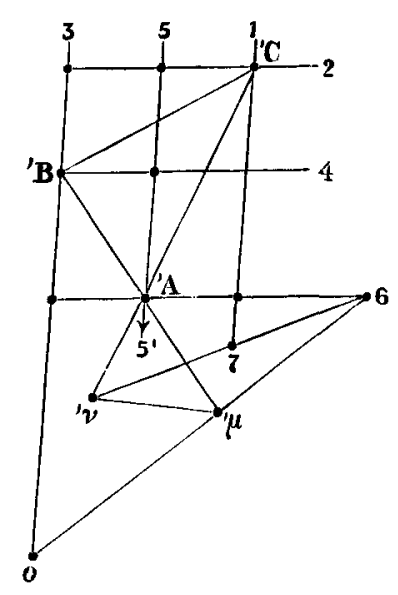

Fig. 3 .

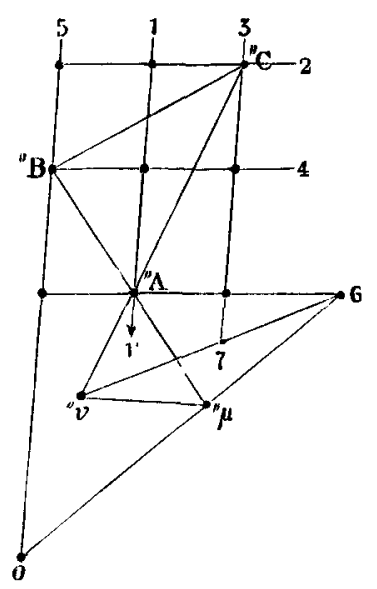

Fig. 4. 
Man ersieht nun, dass in Fig. $2(A B)$ mit (06) einen Punkt $\mu$, und $(A C)$ mit (76) einen Punkt $\nu$ gemein haben, und dass man $\mu$ mit (o6, 3'34) und $\nu$ mit $\left(76,3^{\prime} 3^{2}\right)$ bezeichnen kann. Entsprechendes findet an den beiden andern Figuren statt.

Die Ebene (o76) wird also

$$
\text { von der Ebene } H \text { in der Linie } \mu \nu \text { geschnitten, }
$$

worin

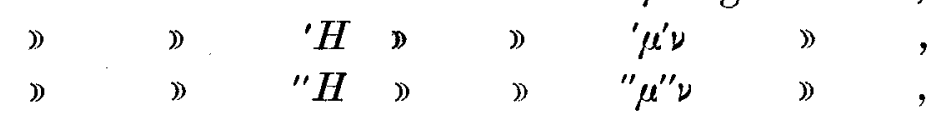

$$
\mu=\left(06,3^{\prime} 34\right), \quad ' \mu=(\text { o6, 5'54), } " \mu=(\text { o6, I', I } 4)
$$

drei Punkte der Linien (06) sind, und

$$
\nu=\left(76,3^{\prime} 3^{2}\right), \quad{ }^{\prime} \nu=\left(76,5^{\prime} 5^{2}\right), \quad{ }^{\prime} \nu=\left(76, \mathrm{I}^{\prime} \mathrm{I}_{2}\right)
$$

drei Punkte der Linie (76).

Kann ich nun nachweisen, dass das Doppelverhältniss der Punkte $(6, \mu, \mu, " \mu)$ gleich dem Doppelverhältniss der Punkte $(6, \nu, \nu, " \nu)$ ist, so folgt daraus, dass sich die drei Linien $\mu \nu, \mu^{\prime} \nu, \mu^{\prime \prime} \mu$ in einem Punkte treffen, und dass der Schnittpunkt der drei Ebenen $H, H,{ }^{\prime} H$ in die Ebene (o76) fällt.

Diesen Nachweis führe ich folgendermassen. Es schneiden sich, wie ich zeigen werde

$$
\begin{aligned}
& \text { die Ebenen }\left(\mathrm{I}^{\prime} 2\right),\left(33^{\prime} 2\right),\left(55^{\prime} 2\right) \text { in einer Linie }\left(22^{\circ}\right) \text {, } \\
& 》 \quad\left(\mathrm{II}^{\prime} 4\right),\left(33^{\prime} 4\right),\left(55^{\prime} 4\right) 》 \gg\left(44^{\circ}\right) \text {, }
\end{aligned}
$$

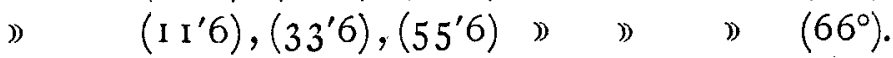

Folglich giebt es ein Hyperboloid, welches die Linien $\left(\mathrm{II}^{\prime}\right),\left(33^{\prime}\right),\left(55^{\prime}\right)$ und die Punkte 2, 4,6 verbindet. Auf diesem lässt sich von 6 aus eine Transversale (66') ziehen, welche mit den Erzeugenden ( $\left.1 \mathrm{I}^{\prime}\right),\left(33^{\prime}\right),\left(55^{\prime}\right)$ zu einem System gehört. Diese Erzeugenden sind aber die Durchschnittslinien entsprechender Ebenen zweier Büschel von gleichem Doppelverhältniss; d. h. die Ebenen

$$
\left(1^{\prime} 2\right),\left(33^{\prime} 2\right),\left(55^{\prime} 2\right),\left(66^{\prime} 2\right)
$$

haben dasselbe Doppelverhältniss wie die Ebenen

$$
\left(\mathrm{II}^{\prime} 4\right),\left(33^{\prime} 4\right),\left(55^{\prime} 4\right),\left(66^{\prime} 4\right) \text {. }
$$


Und hieraus folgt schliesslich, dass, wenn die ersten die Linie (76) in den Punkten (" $\nu \nu ' \nu 6)$, die anderen die Linie (o6) in den Punkten (" $\left.\mu \mu^{\prime} \mu 6\right)$ schneiden, die Doppelverhälnisse beider Punktreihen gleich sein müssen.

Es erübrigt nur noch zu beweisen, dass die Ebenen (I I'2), $\left(33^{\prime} 2\right),\left(55^{\prime} 2\right)$ sich in einer Linie schneiden, [die von (2) ausgeht]. Aus Fig. 2. ist ersichtlich, dass man für $\left(33^{\prime} 2\right)$ auch das Symbol [(3) - (O I 2, 752)] wählen darf. Also ist

$$
\begin{aligned}
& \left(11^{\prime} 2\right)=[(1)-(052,732)], \\
& \left(33^{\prime} 2\right)=\left[(3)-\left(012,75^{2}\right)\right], \\
& \left(55^{\prime} 2\right)=\left[(5)-\left(03^{2}, 712\right)\right] .
\end{aligned}
$$

Die Punkte (o) und (7) kann man durch zwei andere ersetzen, die in der Ebene (I 35) liegen, nämlich durch

Dann hat man

$$
\alpha=(02,135) \text { und } \beta=(72,135) \text {. }
$$

oder wenn man

$$
\begin{aligned}
& \left(\mathrm{II}^{\prime} 2\right)=\left[(2)-(1)-\left(\alpha_{5}, \beta_{3}\right)\right], \\
& \left(33^{\prime} 2\right)=\left[(2)-(3)-\left(\alpha \mathrm{I}, \beta_{5}\right)\right], \\
& \left(55^{\prime} 2\right)=\left[(2)-(5)-\left(\alpha_{3}, \beta_{1}\right)\right],
\end{aligned}
$$

setzt:

$$
\begin{aligned}
& \left(\alpha_{5}, \beta_{3}\right)=a, \\
& \left(\alpha \mathrm{I}, \beta_{5}\right)=b, \\
& \left(\alpha_{3}, \beta_{1}\right)=c
\end{aligned}
$$

$$
\left(1^{\prime} 2\right)=(1 a 2), \quad\left(33^{\prime} 2\right)=(3 b 2) ; \quad\left(55^{\prime} 2\right)=(5 c 2) .
$$

Nun schneiden sich aber die drei Linien $(\mathrm{I} a),(3 b),(5 c)$ in einem Punkte $\left(2^{\circ}\right)$, weil die 6 Linien $(\mathrm{I} \alpha),(\mathrm{r} \beta),(3 \alpha),(3 \beta),(5 \alpha),(5 \beta)$ ein Brianchon'sches Sechseck mit den auf einander folgenden Ecken ( $\left.1 c_{3} a_{5} b\right)$ bilden, folglich haben die Ebenen (I I'2), $\left(33^{\prime} 2\right),\left(55^{\prime} 2\right)$ die Linie $\left(22^{\circ}\right)$ gemein.

Das Ergebniss dieser Betrachtungen ist also der Satz:

„Die drei Ebenen

$$
\begin{aligned}
& H(\text { o } 2.3 .457 .6), \\
& H(\text { O32.5.4 I } 7.6), \\
& H\left(\mathrm{O}_{2} .1 .437 .6\right)
\end{aligned}
$$

schneiden sich in einem Punkte $\Sigma$ der Ebene $(670) .0$ 
Über die acht Schnittpunkte dreier Oberflichen zweiter Ordnung.

Man erkennt leicht, dass die Ebene $H\left(\mathrm{OI}_{2} \cdot 3.457 .6\right)$ noch durch drei andere Punkte $\Sigma$ geht, denn man kann die Ziffern I, 3, 5, die cyklisch zu vertauschen sind, durch 035, I 37 oder 037 ersetzen.

Jede $H$-Ebene enthält also 4 Punkte $\Sigma$.

Die Zahl der Punkte $\Sigma$ ist 5040.4:3=6720.

In jeder durch drei von den Punkten $0,1 \ldots 7$ bestimmten Ebene liegen 120 Punkte $\Sigma^{\prime}$.

\section{$\S 8$.}

Es werde die Ebene $H$ (O 2.3.457.6) polarisirt in Bezug auf die Polartetraeder (0246) und (1 357$)$.

$\mathrm{Zu}\left(\mathrm{O} \mathrm{1}_{2}, 457\right)$ ist die Linie $[(46,357)-(13,026)]$ conjugirt; die Polare von $(23,567)$ ist die Ebene $[(046,157)-(13,024)]$, d. i. $H(046.3 .157 .2)$; die Polare von $(34,016)$ die Ebene [(026, I 57)-- $(24,357)]$, d. i. $H(062 \cdot 4 \cdot 157 \cdot 3)$.

Daraus folgt:

》Die Linie $A=[(46,357)-(\mathrm{I} 3,026)]$ trifft den Durchschnitt

der beiden Ebenen $H(046.3 .157 .2)$ und $H(062.4 .157 .3)$ in einem Punkte $K$.)

Berücksichtigt man, dass durch $\Lambda 4 H$-Ebenen hindurchgehen $(\S 3)$, so hat man den Satz:

„Die sechs Ebenen

$$
\begin{aligned}
& H\left(\mathrm{o} 62.4 . \mathrm{I}_{57.3}\right), H(064 . \mathrm{I} .357 .2), H(264 . \mathrm{I} .357 .0), \\
& H(\mathrm{O} 46.3 .157 .2), H(\mathrm{O} 26.4 . \mathrm{I} 37.5), H(\mathrm{O} 26.4 . \mathrm{I} 35.7)
\end{aligned}
$$

schneiden sich in einem Punkte $K$.»

Liegen die Punkte I, 2 .6 in einer Ebene $E$, so haben mit ihr die $H$-Ebenen der ersten Vertikalreihe die Pascal'schen Linien

$$
P\left(624{ }^{1} 53\right) \text { und } P(463 \text { I52) }
$$

gemein, jede der beiden $H$-Ebenen der zweiten Reihe die Pascal'sche Linie $P\left(64^{1} 35^{2}\right)$, während die letzten zwei $H$-Ebenen mit ihr zusammenfallen. Aus unserem Satz folgt also für die ebene Figur, dass sich 
odie drei Pascal'schen Linien

$$
P(624153), P(463152), P(641352)
$$

in einem Punkte $K$ schneiden,»

Dieser Punkt ist bekanntlich ein Kirkman'scher Punkt; in der rüumlichen Figur werden wir also $K$ als das Analogon zu einem Kirkman'schen Punkte ansehen. ${ }^{1}$

$K$ ist definirt durch das Symbol

$$
K\left\{\begin{array}{c}
(64,357)-(\mathrm{I} 3,260) \\
H(062.4 .157 .3) \\
H(046.3 .157 .2)
\end{array}\right\}
$$

Vertauscht man hierin 1) $\circ$ mit 2, 2) o, 1, 2, 3 mit resp. 5, 4, 7,6 und 3) o, 1, 2, 3 mit resp. 7, 4, 5, 6, so erhält man drei andere Punkte $K$, die aber alle auf derselben Linie $\Lambda$ liegen. Jede Linie $\Lambda$ enthalt also 4 Punkte $K$, und

»die Zahl der Punkte $K$ ist 5040.4=20160.»

Wir suchen nun die Zahl derjenigen Permutationen der Ziffern o, I...7, welche in $K$ das Symbol einer der $H$-Ebenen unverandert lassen.

Zunächst setzen wir in $K$ für $4,6,3,2$ resp. $6,2,4,3$ und finden

$$
K_{1}\left\{\begin{array}{c}
(26,457)-(14,320) \\
H(023.6 .157 .4) \\
H(062.4 .157 .3)
\end{array}\right\}
$$

Vertauschen wir dann in $K$ sowohl, wie in $K_{1}$ I) o mit 6, 2) 1, 5, 7 mit resp. 2,6, o und 3) I , 5,7 mit 2, 0, 6, so bleibt das Symbol $H(062.4 \cdot 157 \cdot 3)$ unverăndert und wir schliessen:

1 Die Methode dieses Paragraphen ist eine Nachbildung derjenigen, vermittelst welcher G. Bauer die teilweise Polarität zwischen Pascal'schen Linien und Kirkman'schen Punkten nachgewiesen hat. (Abh. d. Bayer. Akad. d. Wissensch., Bd. I I, p. I II - I 39. I 874.) 
»In jeder $H$-Ebene liegen 8 Punkte $K$, die keiner ihrer $A$ Linien angehören.»

Uberdies hat jede $H$-Ebene 4 Linien $A$ mit je vier Punkten $K$, folglich enthält jede $H$-Ebene im ganzen 24 Punkte $K$, und, da durch jeden Punkt $K 6 H$-Ebenen gehen, ist 5040.24:6=20160 die Zahl der überhaupt vorhandenen Punkte $K$.

\section{$\S 9$.}

Durch cyklische Vertauschung der Ziffern 2, 4,6 findet man aus

$$
\begin{gathered}
K\left\{\begin{array}{c}
(64,357)-(13,260) \\
H(062.4 \cdot 157.3) \\
H(046.3 \cdot 157.2)
\end{array}\right\} \\
K^{\prime}\left\{\begin{array}{c}
(26,357)-(13,420) \\
H(024.6 .157 .3) \\
H(062.3 .157 .4)
\end{array}\right\}
\end{gathered}
$$

und

$$
K^{\prime \prime}\left\{\begin{array}{c}
(42,357)-(\mathrm{I} 3,640) \\
H(046.2 \cdot \mathrm{I} 57 \cdot 3) \\
H(024 \cdot 3 \cdot 157.6)
\end{array}\right\}
$$

$K$ war der Pol von $H$ (OI 2.3.457.6) in Bezug auf die Polartetraeder (0246) und (1357); $K^{\prime}$ und $K^{\prime \prime}$ sind mithin in demselben Polarsystem die Pole von $H^{\prime}$ (OI 4.3.657.2) und $H^{\prime \prime}$ (OI6.3.257.4). Da aber $H, H^{\prime}, H^{\prime \prime}$ sich in einer Linie schneiden $(\$ 6)$, so folgt:

"Die drei Punkte $K, K^{\prime}, K^{\prime}$ liegen auf einer Geraden $C_{. »}$

Diese Linie $C$ entspricht einer (Salmon-) Cayley'schen Linie der Pascal'. schen Figur.

Die Zahl der Linien $C$ ist $\mathrm{I} 680.4=6720$, denn jeder Linie $S t$ entsprechen vier Linien $C$, weil man als erstes Polartetraeder jedes der vier folgenden (0246), (1 246), (5246), (7246) wählen kann. 
§ IO.

In $\$ 7$ ist nachgewiesen worden, dass jede durch drei der Punkte O, I . . . 7 bestimmte Ebene I 20 Punkte enthält, die Steiner'schen Punkten der Pascal'schen Figur entsprechen. Es lässt sich nun zeigen, dass in jeder dieser Ebenen auch solche Punkte liegen, die Kirkman'schen Punkten entsprechen.

Ich ziche in der Ebene (346) drei gerade Linien, I) die Linie (34), 2) den Durchschnitt (OI 6,346) und 3) die Spur der Ebene H(O 6.3.427.5), d. i. die Linie, welche durch die Punkte $(36,275)$ und $(34$, o I 5) geht.

Diese Linien 1), 2), 3) bilden ein Dreieck $\Delta$; in Verbindung mit demselben betrachte ich das Dreieck $\Delta^{\prime}$ mit den Seiten

$$
\text { I') } \left.\left.^{\prime}(\text { O I } 2,346), \quad 2^{\prime}\right)(457,346), \quad 3^{\prime}\right) \quad(36) \text {. }
$$

Die Seiten I) und I') schneiden sich in dem Punkte (34, o I 2), die Seiten 2) und $2^{\prime}$ ) in dem Punkte (OI6, 457, 346), schliesslich die Seiten 3) und $\left.3^{\prime}\right)$ in dem Punkte $(36,275)$. Diese drei Punkte liegen aber auf dem Durchschnitt der Ebene $H$ (or6.3.457.2) mit (346), folglich müssen die Linien, welche die entsprechenden Ecken der Dreiecke $\Delta$ und $\Delta^{\prime}$ verbinden, durch einen und denselben Punkt gehen.

Die Ecke $(I, 2)$ in $\Delta$ ist der Punkt $\left(34\right.$, OI6), die Ecke $\left(I^{\prime}, 2^{\prime}\right)$ in $d^{\prime}$ der Punkt (OI $2,457,346$ ) und ihre Verbindungslinie der Durchschnitt von $H(012.3 .457 .6)$ mit (346).

Die Ecke $(2,3)$ in $A$ ist der Punkt $($ or $6,427,346)$, die Ecke $\left(2^{\prime}, 3^{\prime}\right)$ in $J^{\prime}$ der Punkt $(36,457)$ und ihre Verbindungslinie der Durchschnitt von $H$ (or6.3.247.5) mit (346).

Da schliesslich die Ecken ( 1,3$)$ und $\left(\mathrm{I}^{\prime}, 3^{\prime}\right)$ in die Punkte $(34$, o I 5 ) und (36, or 2) fallen, so können wir den Satz aussprechen:

)Die Verbindungslinie der Punkte (34, O I 5) und (36, o I 2) trifft

den Durchschnitt der Ebenen $H(\mathrm{OI} 2.3 .457 .6)$ und $H$ (OI6.3.247.5)

in einem Punkte $x$ ),

oder, wenn man noch die $H$-Ebenen berücksichtigt, die durch die beiden $V$-Punkte gehen, in anderer Form: 
Über die acht Schnittpunkte dreier Oberflichen zweiter Ordnung.

»Die vier Ebenen

$$
\begin{aligned}
& H(\text { o } 2.3 .457 .6), H(\text { o } 5.6 .347 .2), \\
& H(\text { O } 6.3 .247 .5), H(\text { OI } 2.4 .367 .5)
\end{aligned}
$$

schneiden sich in einem Punkte $x$ der Ebene (346).)

Es ist leicht zu zeigen, dass im Grenzfalle der Punkt $\%$ in einen Kirkman'schen Punkt übergeht.

\section{$\S \mathrm{I}$ I.}

Zum Schluss vervollständigen wir die Resultate, die mit Hilfe der in $\$ 5$ u. f. f. angewandten Methode zu erlangen sind.

Die acht Punkte o, I ..7 7 lassen sich auf 35 Arten in zwei Gruppen von je vier ordnen. Es giebt also überhaupt 35 Polarsysteme. Sucht man in jedem dieser Systeme den Pol einer und derselben $H$-Ebene, so erhält man 35 Punkte, die sich aber in Io Gruppen von Punkten wesentlich gleichen Characters ordnen lassen. In $\$ 5$ haben wir Repräsentanten von 4 Gruppen, und in $\S 8$ einer fünften Gruppe kennen gelernt. Von den fünf übrigen Gruppen enthält die eine nur V-Punkte als Pole von $H$-Ebenen.

Polarisirt man nämlich $H(\mathrm{OI2.3.456.7)}$ in Bezug auf (OI23) und (4567), so findet man als reciproke Polare von (OI 2, 457) die Linie (37), als Polare von $(23,567)$ die Ebene (or 4$)$ und als Polare von $(34$, or 7 ) die Ebene $\left[\left(\mathrm{OI}_{2}, 567\right)-(23,456)\right]$. Der gesuchte Pol ist also der Punkt $(37,014)$, der in der That mit $(012,567)$ und $(23,456)$ in einer Ebene liegt, nämlich in $H$ (OI $\left.2 \cdot 3 \cdot 75^{6.4}\right)$.

a) Ferner sei $H(\mathrm{O} 12.3 .456 .7)$ zu polarisiren in Bezug auf (OI57) und (2346). Die reciproke Polare von $(012,456)$ ist die Linie

$$
[(57,346)-(23, \text { O } 77)] \text {, }
$$

die Polare von $(34$, o 7$)$ die Ebene (265) und die Polare von $(23,567)$ die Ebene $[(46)-(\mathrm{OI}, 234)]$. Da nun die beiden letztgenannten Ebenen die Punkte (6) und $(401,234,256)$ gemein haben, so besteht der Satz: 
»Die vier Punkte

$$
\text { (6), (OI } 4,234,256),(57,346),(23, \text { O I } 7)
$$

liegen in einer und derselben Ebene.)

Man kann den Satz auch wie folgt bewiesen. Auf der Linie $(657,346)$, welche die Punkte (6) und $(57,346)$ verbindet, liegt auch der Punkt $(34,567)$. Durch diesen und durch den Punkt $(23$, o I 7$)$ gehen aber die Ebenen $H$ (OI 4.3.256.7) und (234), folglich liegt auf ihrer Verbindungslinie der Punkt

$$
\text { (OI } 4,256,234) \text {. }
$$

b) Es seien (2347) und (OI56) die Tetraeder und $H$ (o 2.3 .456 .7 ) wiederum die Ebene, deren Pol bestimmt werden soll. Die reciproke Polare von $\left(\mathrm{OI} 2,45^{6}\right)$ ist der Linie $[(56,347)-(\mathrm{OI}, 237)]$, die Polare von $(23,567)$ die Ebene $[(47)$ - (O I , 234)] oder, was dasselbe ist, die Ebene $[(7)-($ o I 4,234$)]$, die Polare von $(34$, or 7$)$ die Ebene $[(7)-(256,234)]$. Es muss also der Durchschnitt der beiden Ebenen $[(7)-(014,234)]$ und $[(7)-(256,234)]$ mit den beiden Punkten $(56,347)$ und (01, 237) in einer und derselben Ebene liegen. Da aber dieser Durchschnitt durch die Punkte (7) und (OI 4, 234, 256) bestimmt ist, so hat man den Satz: »Die vier Punkte

$$
\text { (7), (O. } 4,234,256),(56,347),(\text { or }, 237)
$$

liegen in einer und derselben Ebene.)

Dieser Satz lässt sich auch folgendermassen beweisen.

Die beiden von (7) ausgehenden Linien $(765,347)$ und $(710,723)$ bestimmen eine Ebene, welche die drei Punkte

$$
\text { (7), }(56,347),(\mathrm{OI}, 237)
$$

enthalt. Auf der ersten Linie liegt aber der Punkt $(34,567)$, und auf der zweiten der Punkt (23, O 7 7). Durch diese beiden gehen die Ebenen $H$ (OI 4.3.265.7) und (234), folglich liegt auf ihrer Verbindungslinie auch der Punkt

$$
\text { (OI4, 265, 234). }
$$

c) Polarisirt man drittens $H$ (OI2.3.456.7) in Bezug auf (OI34) und (2567), so findet man: 
Ober die acht Schnittpunkte dreier Oberflächen zweiter Ordnung.

)Die Punkte $(34,567)$ und $(27$, o 3$)$ liegen mit dem Durchschnitt der beiden Ebenen $[(2)-($ I I 4,567$)]$ und $[($ O I $)-(34,256)]$ in einer Ebene.)

d) Zum Schluss suche ich noch den Pol von $H(047.3 .126 .5)$ in Bezug auf die Polartetraeder (OI27) und (3456). Die reciproke Polare von $\left(047, \mathrm{I}_{26} 6\right)$ ist die Linie $\left[\left(\mathrm{I}_{2}, 356\right)-(07,345)\right]$, die Polare von $(37,256)$ die Ebene $[(012,456)-(34,701)]$ d. i. $H($ OI 2.3.456.7) und die Polare von $(13,045)$ die Ebene $[(702,456)--(36,127)]$ d. i. $H(720.3 .654 . \mathrm{I})$. Der Durchschnitt von $H($ o 2 2.3.456.7) und $H(720.3 .654 . \mathrm{I})$ liegt also mit den Punkten $(12,356)$ und $(07,345)$ in einer Ebene.

Berücksichtigt man, welche $H$-Ebenen durch diese Punkte gehen, so gelangt man zu dem Satz:

»Die sechs Ebenen

$$
\begin{aligned}
& H\left(\text { O } 2.3 .45^{6.7}\right), H(120.7 .635 .4), H(071.2 .435 .6) \text {, } \\
& H(720.3 .645 .1), H(127.0 .635 .4), H(072.1 .435 .6)
\end{aligned}
$$

schneiden sich in einem Punkte.» 